\title{
Know Yourself: An Adaptive Causal Network Model for Therapeutic Intervention for Regaining Cognitive Control
}

\author{
Nimat Ullah ${ }^{(凶)}(1)$ and Jan Treur $(\mathbb{C}$ \\ Social AI Group, Vrije Universiteit Amsterdam, Amsterdam, The Netherlands \\ nimatullah09@gmail.com, j.treur@vu.nl
}

\begin{abstract}
Long term stress often causes depression and neuronal atrophies that in turn can lead to a variety of health problems. As a result of these cellular changes, also molecular changes occur. These changes, that include increase of glucocorticoids and decrease of the brain-derived neurotrophic factor, have the unfortunate effect that they decrease the cognitive abilities needed for the individual to solve the stressful situation. Such cognitive abilities like reappraisal and their adaptation mechanisms turn out to be substantially impaired while they are needed for regulation of the negative emotions. However, antidepressant treatments and some other therapies have proved to be quite effective for the strengthening of such cognitive abilities. This study introduces an adaptive causal network model for this phenomenon where a subject loses his or her cognitive abilities (negative metaplasticity) due to long-term stress and re-improve these cognitive abilities (positive metaplasticity) through mindfulness-based cognitive therapy (MBCT). Simulation results have been reported for demonstration of the phenomenon.
\end{abstract}

Keywords: Adaptive causal modeling - Negative metaplasticity $\cdot$ Positive metaplasticity $\cdot$ Mindfulness $\cdot$ Cognition $\cdot$ Therapy $\cdot$ Reappraisal

\section{Introduction}

Potentially, there can be various reasons and forms of cognitive alteration such as decline in cognitive abilities with age where the rate of decline varies from person to person (Verhaeghen 2011). The decline can be due to long-term stress (Garcia 2002) or due to reduction of flexibility as the person grows older (Charles 2010). This paper, specifically focuses on the consequences caused by long-term stress. Stress has become one of the most common negative emotion these days. Various studies have found that long-term stress can have very severe consequences (Garcia 2002; Mazure et al. 2002; Tennant 2001). These studies highlight the cognitive decline as one of the main consequences of long-term stressors. Decrease in the brain-derived neurotrophic factor (BDNF) as a result of increase in the glucocorticoids was found to be the main reason of cell loss and hence the alteration in synaptic plasticity at a cellular level (Fuchs and Gould 2000; Sapolsky 1999).

(C) IFIP International Federation for Information Processing 2020

Published by Springer Nature Switzerland AG 2020

I. Maglogiannis et al. (Eds.): AIAI 2020, IFIP AICT 584, pp. 334-346, 2020.

https://doi.org/10.1007/978-3-030-49186-4_28 
However, there are a number of studies like (Garland et al. 2009; Garland et al. 2011) who put forward that mindfulness-based cognitive therapy (MBCT) combining different techniques from therapies and training helps in strengthening cognitive abilities, specifically those impaired by long-term stress. It works by focusing on present moment, gaining awareness of one's self and accepting the reality. This study considers MBCT instead of standard CBT, being one of the most studied therapies, due to the fact that some studies like (Troy et al. 2013) have compared MBCT and CBT in terms of their performance and found that MBCT is more effective.

Moreover, this study considers an adaptive causal network modeling approach (Treur 2020) to model the above mentioned phenomena because stressful emotions and their effects form an adaptive and cyclic process which this approach particularly handles quite effectively as demonstrated, for example, in (Ullah and Treur 2020; Ullah and Treur 2019). This modeling approach can be considered as a branch in the causal modeling area which has a long tradition in AI; e.g., see (Kuipers 1984; Kuipers and Kassirer 1983; Pearl 2009). It distinguishes itself by a dynamic perspective on causal relations, according to which causal relations exert causal effects over time, and in addition these causal relations themselves can change over time as well. The basic type of network model used is called a multilevel adaptive temporal-causal network model. By adding dynamics an adaptation to causal modeling, applications become possible that otherwise would be out of reach of causal modeling. This provides a useful approach to translate (supported by a dedicated modeling environment) qualitative processes as known from empirical literature into adaptive causal network models that can be used for simulation.

In the rest of the paper, Sect. 2 gives brief account of the literature on the subject, Sect. 3 presents the adaptive cognitive network model which is explained by simulation results in detail in Sect. 4. Finally, the paper is concluded in Sect. 5.

\section{Background Literature}

Research on stress, being one of the common negative emotions, has come up with vital long lasting negative consequences of it (Mazure et al. 2002; Tennant 2001). Studies like (Garcia 2002) have shown its contribution to depression and the adaptive casual way it affects one's cognitive abilities. It's worth noting here that the structural and functional changes brought about by stress are similar to those of depression. At a cellular level, stress and depression cause cell and neuronal losses. By (Sapolsky 1999) such cellular changes in the hippocampus are linked to the increase in plasma levels of glucocorticoid hormones like cortisol.

At a molecular level, the cellular deficiencies were found to take place at the hippocampus. This happens most of the time due to the decrease in the expression of the brain-derived neurotrophic factor (BDNF) associated with elevation of glucocorticoids (Fuchs and Gould 2000; Sapolsky 1999). Similarly, (Smith et al. 1995) also support the notion that high levels of glucocorticoids induced by stress and its administration are considered to be down-regulating the hippocampal expression. (Mocchetti et al. 1996) considers the reduction of BDNF, which supports neuronal survival and function in the hippocampus, as the potential mediating action of glucocorticoid on hippocampus. The 
effect of the boost of glucocorticoids is referred to as negative metaplasticity as it downregulates adaptivity of the hippocampal synaptic connectivity. In contrast, the boost in the expression of BDNF is referred to as positive metaplasticity as it strengthens adaptivity of the connectivity in the hippocampus. As a result of these changes, the subject loses his control of cognitive abilities and is unable to efficiently regulate his emotions in an adaptive manner. On the other hand, the same mechanisms can reversibly be used via treatment as well (Garcia 2002), i.e., through the increase in the expression of BDNF. Synapses are responsible for the processing and transmission of neural information with some efficacy and their alteration is referred to as 'synaptic plasticity', which is a form of (first-order) adaptation. The mechanisms described above indicate how synaptic plasticity itself also can change, which is a form of second-order adaptation usually called metaplasticity. When metaplasticity enhances the adaptive cognitive function, by Garcia (2002) it is called positive metaplasticity and if this change brings some impairment to the adaptive cognitive function, then it's referred to as negative metaplasticity this indeed has been observed in case of long-term stress (Foster 1999; Kim and Yoon 1998); similar cognitive impairment has been observed in various other studies in both humans (Lupien et al. 1997) and animals (Mizoguchi et al. 2000).

Cognitive reappraisal, as a strategy, is considered to be a very adaptive strategy which contributes to positive psychological health by reducing negative effects and it is associated to improved memory and interpersonal functioning in contrast to, for example, suppression (Butler et al. 2003; Gross and John 2003; John and Gross 2004).

To deal with the cognitive decline caused by long-term stressors, we consider Mindfulness-based cognitive therapy (MBCT) as found quite effective by various researchers in this domain (Garland et al. 2009; Garland et al. 2011). The purpose of MBCT is to improve psychological health by increasing mindfulness. This therapy combines Kabat-Zinn's (Kabat-Zinn 1990) mindfulness-based stress reduction program with the techniques used in Cognitive Behavior Therapy (CBT). Kabat-Zinn's MindfulnessBased stress reduction program involves daily meditation and self-awareness exercises. The MBCT training program aims at promoting acceptance of thoughts/feelings without being judgmental, focusing on the present moment and awareness of one's self (Coffey et al. 2010). According to (Allen et al. 2006) practicing of acceptance is meant to develop the ability to distinguish oneself from the contents of negative thoughts and recognize that emotions are non-permanent events. This is achieved by bringing a person in a decentered metacognitive state which may help in increasing cognitive flexibility through disengagement from the initial negative thoughts/appraisal, as has been considered essential by (Garland et al. 2009). After disengagement comes the ability to successfully switch from negative appraisal to positive appraisal and previous research credits this ability to mindfulness training (Jha et al. 2007). Similarly, MBCT encourages individuals to focus on the present moment as it is expected to give insight into one's own (wide range of) stimuli related physical sensations, feelings and thoughts. Otherwise, chances of failure increase in the reappraisal/re-interpretation of the thoughts without knowing about it.

\section{Multilevel Adaptive Cognitive Modeling}

This section of the paper gives overview of the multilevel adaptive causal network modeling approach (Treur 2016, 2020) that has been used for development and simulation of 
the adaptive causal network model. Table 1 summarizes the main conceptual and numerical representations for this adaptive causal modeling approach. The Network-oriented modeling approach provides a library of over 36 combination functions. Apart from the available combination functions, self-defined combination functions can also be added to the library which makes the approach quite flexible and supports a wide application range.

Table 1. Conceptual and numerical representations for the adaptive causal modeling approach

\begin{tabular}{|c|c|c|}
\hline Concept & Conceptual representation & Explanation \\
\hline $\begin{array}{l}\text { States and } \\
\text { connections }\end{array}$ & $X, Y, X \rightarrow Y$ & $\begin{array}{l}\text { Describes the nodes (representing } \\
\text { states) and links (representing } \\
\text { causal connections between } \\
\text { states) of the network structure }\end{array}$ \\
\hline $\begin{array}{l}\text { Connection } \\
\text { weight }\end{array}$ & $\omega_{X, Y}$ & $\begin{array}{l}\text { The connection weight } \omega_{X, Y} \in \\
{[-1,1] \text { represents the strength of }} \\
\text { the causal impact of state } X \text { on } \\
\text { state } Y \text { through connection } X \rightarrow Y\end{array}$ \\
\hline $\begin{array}{l}\text { Aggregating } \\
\text { multiple } \\
\text { impacts on a } \\
\text { state }\end{array}$ & $\mathbf{c}_{Y}(.)$. & $\begin{array}{l}\text { For each state } Y \text { (a reference to) a } \\
\text { combination function } \mathbf{c}_{Y}(. .) \text { is } \\
\text { chosen to combine the causal } \\
\text { impacts of other states on state } Y\end{array}$ \\
\hline $\begin{array}{l}\text { Timing of } \\
\text { the effect of } \\
\text { causal } \\
\text { impact }\end{array}$ & $\eta_{Y}$ & $\begin{array}{l}\text { For each state } Y \text { a speed factor } \\
\eta_{Y} \geq 0 \text { is used to represent how } \\
\text { fast a state is changing upon } \\
\text { causal impact }\end{array}$ \\
\hline Concept & Numerical representation & Explanation \\
\hline $\begin{array}{l}\text { State values } \\
\text { over time } t\end{array}$ & $Y(t)$ & $\begin{array}{l}\text { At each time point } t \text { each state } Y \\
\text { in the model has a real number } \\
\text { value in }[0,1]\end{array}$ \\
\hline $\begin{array}{l}\text { Single } \\
\text { causal } \\
\text { impact }\end{array}$ & $\begin{array}{l}\operatorname{impact}_{X, Y}(t) \\
=\omega_{X, Y} X(t)\end{array}$ & $\begin{array}{l}\text { At } t \text { state } X \text { with a connection to } \\
\text { state } Y \text { has an impact on } Y \text {, using } \\
\text { connection weight } \omega_{X, Y}\end{array}$ \\
\hline $\begin{array}{l}\text { Aggregating } \\
\text { multiple } \\
\text { causal } \\
\text { impacts }\end{array}$ & $\begin{array}{l}\operatorname{aggimpact}_{Y}(t) \\
=\mathbf{c}_{Y}\left(\operatorname{impact}_{X 1, Y}(t), \ldots, \operatorname{impact}_{X k, Y}(t)\right) \\
=\mathbf{c}_{Y}\left(\boldsymbol{\omega}_{X 1, Y} X_{1}(t), \ldots, \boldsymbol{\omega}_{X k, Y} X_{k}(t)\right)\end{array}$ & $\begin{array}{l}\text { The aggregated causal impact of } \\
\text { multiple states } X_{i} \text { on } Y \text { at } t \text {, is } \\
\text { determined using combination } \\
\text { function } \mathbf{c}_{Y}(. .)\end{array}$ \\
\hline $\begin{array}{l}\text { Timing of } \\
\text { the causal } \\
\text { effect }\end{array}$ & $\begin{array}{l}Y(t+\Delta t)=Y(t)+ \\
\eta_{Y}\left[\operatorname{aggimpact}_{Y}(t)-Y(t)\right] \Delta \mathrm{t} \\
=Y(t)+\eta_{Y}\left[\mathbf{c}_{Y}\left(\boldsymbol{\omega}_{X 1, Y} X_{1}(t), \ldots\right.\right. \\
\left.\left.\boldsymbol{\omega}_{X k, Y} X_{k}(t)\right)-Y(t)\right] \Delta \mathrm{t}\end{array}$ & $\begin{array}{l}\text { The causal impact on } Y \text { is exerted } \\
\text { over time gradually, using speed } \\
\text { factor } \eta_{Y} ; \text { here the } X_{i} \text { are all } \\
\text { states with outgoing connections } \\
\text { to state } Y\end{array}$ \\
\hline
\end{tabular}


Table 2 provides explanation of the various states of the adaptive causal network model proposed in this paper. In the table and the figure, the background colors differentiate between the (adaptation) levels of the model and are part of the meaning; they are

Table 2. Overview of the states of the multi-level network model in Fig. 1

\begin{tabular}{|c|c|c|c|}
\hline & State & Explanation & Level \\
\hline$X_{1}$ & $\mathrm{ws}_{s}$ & World state for stimulus ' $s$ ' & \multirow{17}{*}{ Base level } \\
\hline$X_{2}$ & $\mathrm{SS}_{S}$ & Sensor state for stimulus ' $s$ ' & \\
\hline$X_{3}$ & $\operatorname{srs}_{S}$ & Sensory representation state for stimulus ' $s$ ' & \\
\hline$X_{4}$ & $\mathrm{gs}_{b}$ & Goal state for body ' $b$ ' & \\
\hline$X_{5}$ & $\mathrm{ps}_{g}$ & Preparation state for goal ' $g$ ' & \\
\hline$X_{6}$ & $\mathrm{es}_{g}$ & Execution state for goal ' $g$ ' & \\
\hline$X_{7}$ & $\mathrm{ss}_{\mathrm{b}}$ & Sensor state for body state $b$ & \\
\hline$X_{8}$ & $\operatorname{srs}_{b}$ & Sensory representation state for body sate $b$ & \\
\hline$X_{9}$ & $\mathrm{fs}_{b}$ & Feeling state for body state $b$ & \\
\hline$X_{10}$ & $\mathrm{ps}_{b}$ & Preparation state for body state $b$ & \\
\hline$X_{11}$ & $\mathrm{es}_{b}$ & Expression execution state for body state $b$ & \\
\hline$X_{12}$ & $\mathrm{bs}_{-}$ & Belief state for negative belief - & \\
\hline$X_{13}$ & $\mathrm{bs}_{+}$ & Belief state for positive belief + & \\
\hline$X_{14}$ & $\operatorname{accp}_{b}$ & Acceptance state for body & \\
\hline$X_{15}$ & $a w_{b}$ & Awareness state for body & \\
\hline$X_{16}$ & $\mathrm{pr}_{b}$ & Present moment state of body & \\
\hline$X_{17}$ & $\mathrm{Cs}_{\text {reapp }}$ & Control state for cognitive reappraisal & \\
\hline$X_{18}$ & $\mathbf{W}_{\mathrm{fs}_{b}, \mathrm{cs}_{\text {reapp }}}$ & Reified representation state for connection weight $\omega_{\mathrm{fs} b, \mathrm{~s}_{\text {reapp }}}$ & $\begin{array}{l}\text { First Reification } \\
\text { Level }\end{array}$ \\
\hline$X_{19}$ & $\mathbf{M}_{\mathbf{W}_{\mathrm{fs}_{b^{\prime}}, \mathrm{cs}_{\text {reapp }}}}$ & $\begin{array}{l}\text { Reified representation state for persistence factor } \mu \text { for } \\
\mathbf{W}_{\mathrm{fs}_{b}, \mathrm{cs}_{\text {seapp }}}\end{array}$ & $\begin{array}{l}\text { Second Reifica- } \\
\text { tion Level }\end{array}$ \\
\hline$X_{20}$ & $\mathbf{H}_{\mathbf{W}_{\mathrm{fs}_{p^{\prime}}, \mathrm{cs} \text { reapp }}}$ & Reified representation state for speed factor $\eta$ for $\mathbf{W}_{\mathrm{fs}_{b}, \mathrm{cs}_{\text {reapp }}}$ & \\
\hline
\end{tabular}

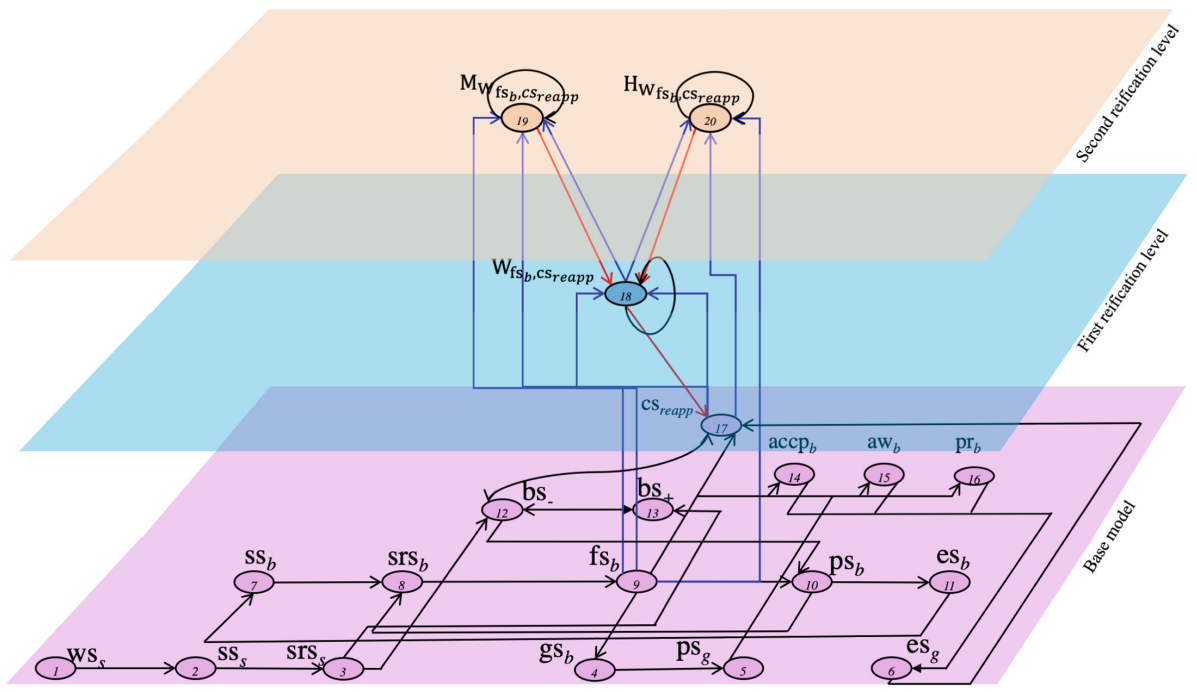

Fig. 1. Adaptive causal network model for therapeutic intervention for long-term stress 
called reification levels as they explicitly represent by their states some of the network characteristics of the level below. For instance, the base model has 17 states, the first reification level modeling synaptic plasticity (first-order adaptation) has 1 state and the second reification level modeling metaplasticity (second-order adaptation) has 2 states. The modeled synaptic plasticity at the first reification level is addressed by the (adaptive) connection weight $\omega_{\mathrm{fs}_{b}}$, cs reapp by representing it by reification state $\mathbf{W}_{\mathrm{fs}_{b}}, \mathrm{cs}_{\text {reapp }}$. The metaplasticity concerning adaptation of the dynamics of the adaptation of this connection weight is addressed by the second-level reification states $\mathbf{M}_{\mathbf{W}_{\mathrm{fs}_{b}}, \mathrm{cs}_{\text {reapp }}}$ for the persistence of the learnt effects and $\mathbf{H}_{\mathbf{W}_{\mathrm{fs}_{b}} \text {, cs reapp }}$ for the speed of the adaptation process.

The connectivity picture of the model shown in Fig. 1 demonstrates the phenomenon where long-term stress causes negative metaplasticity of cognitive abilities, i.e. loss in the ability to use and adapt cognitive reappraisal for regulation of negative emotions. In contrast, mindfulness-based cognitive therapy has been used as an intervention to obtain positive metaplasticity through which upregulation of the cognitive reappraisal ability can take place. In Fig. 1, the bottom level represents the base model which performs the basic function of regulation of emotions through reappraisal. Cognitive reappraisal changes one's perception/belief about the stimulus: from a negative to a positive interpretation in this model. Generally, reappraisal can also be from positive believe to negative belief depending upon the demand of the situation, but in this model, reappraisal refers to the reinterpretation to positive belief from negative. The first reification level modeling first-order adaptation, represents Hebbian learning for the connection from base state $\mathrm{fs}_{b}$ to base state $\mathrm{cs}_{\text {reapp }}$. The dynamics of this state $\mathbf{W}_{\mathrm{fs}_{b}}$, $\mathrm{cs}_{\text {reapp }}$ represents the learning taking place for this connection at the base level. Learning doesn't only refer to increase in the strength of the connection, it can also involve decrease in the strength of the connection over time. In this model, as demonstrated in Figs. 2 and 3, initially, this connection from $\mathrm{fs}_{b}$, to $\mathrm{cs}_{\text {reapp }}$ gets weaker due to long-term stress. When this connection gets weaker, the negative (body) feeling state $\mathrm{fs}_{b}$ gets higher which activates the goal state $\mathrm{gs}_{b}$ to address the problem, as also demonstrated in (Mohammadi Ziabari and Treur 2019) for such kind of interventions. This goal state represents the intention of the person to undergo some kind of therapy for getting back on track. In the scenario used for this model, goal activation refers to the intention to undergo MBCT (therapy) but in general it can be any kind of intervention, for example, antidepressant treatment as suggested in (Garcia 2002) etc.

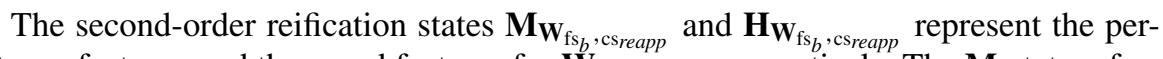
sistence factor $\mu$ and the speed factor $\eta$ for $\mathbf{W}_{\mathrm{fs}_{b}}, \mathrm{cs}_{\text {reapp }}$ respectively. The $\mathbf{M}$-state refers to how long or short the $\mathbf{W}$-state retains its learned value. Similarly, the $\mathbf{H}$-state controls the speed factor of the $\mathbf{W}$-state managing how fast or slow the $\mathbf{W}$-state learns of forgets the value. Change in the speed and persistence factor is referred to as metaplasticity. The upregulation is called positive while the downregulation is called negative metaplasticity.

The two boxes below give insight into the different dynamics of the network. The colors and the boxes itself are part of the meaning and standard defined by network oriented modeling approach in (Treur 2020). In the boxes below, Box 1 represent the role matrices $\mathbf{m b}$ and $\mathbf{m c w}$. In role matrix $\mathbf{m b}$, each state has a row which shows all the incoming connections to that state. It's worth mentioning here that in matrix $\mathbf{m b}$ each state has only the incoming connections which are either at the same level or come 
from a lower level. The downward connections are indicated in the other role matrices instead. For instance, in matrix mow the state number $X_{18}$ (i.e., the $\mathbf{W}$-state) represents the adaptive connection from $X_{9}$ to $X_{17}$ at the base level, the causal effect of which is modeled by the downward connection from $X_{18}$. Moreover, the values between -1 and 1 in role matrix mcw represent the connection weights of the incoming connections to that specific state. The specific values in the role matrices allow to get the simulation pattern shown in Fig. 2 and Fig. 3. The values were chosen such that the simulation results exhibit a pattern similar to the ones found in the literature.

In Box 2, role matrices mcfw, mcfp and $\mathbf{m s}$ are given. Matrix $\mathbf{m c f w}$ indicates the combination function used at a state $X_{i}$ for the aggregation of incoming causal impact from other states to state $X_{i}$. For instance, state $X_{1}$ uses the identify function, state $X_{18}$ uses the Hebbian combination function and state $X_{19}$ uses alogistic(..) as combination function, defined by

$$
\operatorname{alogistic}_{\boldsymbol{\sigma}, \boldsymbol{\tau}}\left(V_{1}, \ldots, V_{k}\right)=\left[\frac{1}{1+e^{-\sigma\left(V_{1}+\cdots V_{k}-\tau\right)}}+\frac{1}{1+e^{\sigma \tau}}\right]\left(1+e^{-\sigma \tau}\right)
$$

Moreover, the first-order adaptation state $X_{18}$ uses the Hebbian learning combination function hebb(..) defined by

$$
\operatorname{hebb}_{\mu}\left(V_{1}, V_{2}, W\right)=V_{1} V_{2}(1-W)+\boldsymbol{\mu} W
$$

\begin{tabular}{|c|c|c|c|c|c|c|c|c|c|c|c|}
\hline \multicolumn{2}{|c|}{$\begin{array}{l}\text { mb connectivity: } \\
\text { base connectivity }\end{array}$} & 1 & 2 & 3 & 4 & \multicolumn{2}{|c|}{$\begin{array}{l}\text { mcw connectivity: } \\
\text { connection weights }\end{array}$} & 1 & 2 & \multirow[t]{2}{*}{3} & 4 \\
\hline$X_{1}$ & $\mathrm{ws}_{s}$ & $X_{I}$ & & & & $X_{1}$ & $\mathrm{ws}_{s}$ & 1 & & & \\
\hline$X_{2}$ & $\mathrm{SS}_{s}$ & $X_{1}$ & & & & $X_{2}$ & $\mathrm{ss}_{s}$ & 1 & & & \\
\hline$X_{3}$ & $\operatorname{srs}_{S}$ & $X_{2}$ & & & & $X_{3}$ & $\operatorname{srs}_{S}$ & 1 & & & \\
\hline$X_{4}$ & $\mathrm{gs}_{b}$ & $X_{9}$ & & & & $X_{4}$ & $\mathrm{gs}_{b}$ & 0.8 & & & \\
\hline$X_{5}$ & $\mathrm{ps}_{g}$ & $X_{4}$ & & & & $X_{5}$ & $\mathrm{ps}_{g}$ & 1 & & & \\
\hline$X_{6}$ & $\mathrm{es}_{g}$ & $X_{14}$ & $X_{15}$ & $X_{16}$ & & $X_{6}$ & $\mathrm{es}_{g}$ & 0.5 & 0.5 & 0.58 & \\
\hline$X_{7}$ & $\mathrm{Ss}_{\mathrm{b}}$ & $X_{11}$ & & & & $X_{7}$ & $\mathrm{ss}_{\mathrm{b}}$ & 1 & & & \\
\hline$X_{8}$ & $\operatorname{srs}_{b}$ & $X_{7}$ & $X_{10}$ & & & $X_{8}$ & $\operatorname{srs}_{b}$ & 0.27 & 0.3 & & \\
\hline$X_{9}$ & $\mathrm{fs}_{b}$ & $X_{8}$ & & & & $X_{9}$ & $\mathrm{fs}_{b}$ & 1 & & & \\
\hline$X_{10}$ & $\mathrm{ps}_{b}$ & $X_{9}$ & $X_{12}$ & & & $X_{10}$ & $\mathrm{ps}_{b}$ & 0.1 & 0.45 & & \\
\hline$X_{11}$ & $\mathrm{es}_{b}$ & $X_{10}$ & & & & $X_{11}$ & $\mathrm{es}_{b}$ & 1 & & & \\
\hline$X_{12}$ & $\mathrm{bs}_{-}$ & $X_{3}$ & $X_{13}$ & $X_{17}$ & & $X_{12}$ & $\mathrm{bs}_{-}$ & 0.6 & -0.4 & -0.21 & \\
\hline$X_{13}$ & $\mathrm{bs}_{+}$ & $X_{3}$ & $X_{12}$ & & & $X_{13}$ & $\mathrm{bs}_{+}$ & 0.4 & -0.35 & & \\
\hline$X_{14}$ & $\operatorname{accp}_{b}$ & $X_{5}$ & $X_{9}$ & & & $X_{14}$ & $\operatorname{accp}_{b}$ & 0.9 & 0.2 & & \\
\hline$X_{15}$ & $a w_{b}$ & $X_{5}$ & $X_{9}$ & & & $X_{15}$ & $a w_{b}$ & 0.9 & 0.2 & & \\
\hline$X_{16}$ & $\mathrm{pr}_{b}$ & $X_{5}$ & $X_{9}$ & & & $X_{16}$ & $\mathrm{pr}_{b}$ & 0.9 & 0.2 & & \\
\hline$X_{17}$ & $\mathrm{cs}_{\text {reapp }}$ & $X_{6}$ & $X_{9}$ & $X_{12}$ & & $X_{17}$ & $\mathrm{CS}_{\text {reapp }}$ & 1 & $X_{18}$ & 0.2 & \\
\hline$X_{18}$ & $\mathbf{W}_{\mathrm{fs}_{b^{\prime}} \mathrm{cs}_{\text {reapp }}}$ & $X_{9}$ & $X_{17}$ & $X_{18}$ & & $X_{18}$ & $\mathbf{W}_{\mathrm{fs}_{b^{\prime}} \mathrm{cs}_{\text {reapp }}}$ & 1 & 1 & 1 & \\
\hline$X_{19}$ & $\mathbf{M}_{\mathbf{W}_{\mathrm{fs}_{b^{\prime}} \mathrm{cs} \text { reapp }}}$ & $X_{9}$ & $X_{17}$ & $X_{18}$ & $X_{19}$ & $X_{19}$ & $\mathbf{M}_{\mathbf{W}_{\mathrm{fs}_{b^{\prime}} \mathrm{cs}_{\text {reapp }}}}$ & -1 & 1 & 1 & 1 \\
\hline$X_{20}$ & $\mathbf{H}_{\mathbf{W}_{\mathrm{fs}_{b}, \mathrm{cs} \text { reapp }}}$ & $X_{9}$ & $X_{17}$ & $X_{18}$ & $X_{20}$ & $X_{20}$ & $\mathbf{H}_{\mathbf{W}_{\mathrm{fs}_{b^{\prime}} \mathrm{cs} \text { reapp }}}$ & -1 & 1 & 1 & 1 \\
\hline
\end{tabular}

Box 1 Role matrices for connectivity 
Role matrix mcfp provides the parameter values for each of the combination function used as indicated in mcfw. In the row for $X_{18}$, in column 2 (for Hebb) of mcfp, $X_{19}$ in the red cell indicates the downward causal connection from the second-order reification state to $X_{18}$. In other words, $X_{19}$ is responsible for the persistence factor of $X_{18}$. Similarly, the $X_{20}$ in the red cell at the row for $X_{18}$ in role matrix ms indicates the downward causal connection from the second-order reification level to the first-order reification level. In other words, $X_{20}$ is responsible for the speed factor of $X_{18}$. These downward causal connections are essential to causally effectuate the adaptation processes modeled as dynamics at the higher levels.

\section{Simulation Results}

This section of the paper explains the simulation results given in Fig. 2 and Fig. 3 obtained from the adaptive causal network model with connectivity depicted in Fig. 1. These results were obtained using the values in Box 1, Box 2, and the initial values serving as inputs to the states as give in Table 3. Moreover, initial values of the states are given below in Table 2. Figure 2 demonstrates the basic phenomenon as discussed in the literature section, where long-term stressors decrease the adaptive cognitive abilities of the subject. The subject decides to undergo MBCT and regains his cognitive abilities. Figure 2 shows the patterns for all the states at the base level. In the figure, it can be seen that initially the control state $\mathrm{cs}_{\text {reapp }}$ for reappraisal gets activated as soon as negative belief bs - gets higher. The control state $\mathrm{cs}_{\text {reapp }}$ changes the beliefs representing the interpretation of the person and, therefore, bs $s_{-}$decreases while bs + increases. But during this time, gradual decrease in the activation level of $\mathrm{cs}_{\text {reapp }}$ can be observed and that's the time when bs $s_{-}$also stays higher and bs + stays lower.

Table 3. Initial values serving as inputs to the states

\begin{tabular}{l|l|l|l|l|l}
\hline State & $\mathrm{ws}_{s}$ & All other base states & $\mathbf{W}_{\mathrm{fs}_{b}, \mathrm{cs}_{\text {reapp }}}$ & $\mathbf{H W}_{\mathrm{fs}_{b}, \mathrm{cs}_{\text {reapp }}}$ & $\mathbf{M W}_{\mathrm{fs}_{b}, \mathrm{cs}_{\text {reapp }}}$ \\
\hline Initial value & 1 & 0 & 0.3 & 0.5 & 0.9 \\
\hline
\end{tabular}




\begin{tabular}{|c|c|c|c|c|c|c|c|c|c|c|c|c|}
\hline $\begin{array}{l}\text { mcfw aggregation: } \\
\text { Combination func- } \\
\text { tion weights } \\
\end{array}$ & $\begin{array}{c}1 \\
\text { alo- } \\
\text { gistic }\end{array}$ & $\begin{array}{c}2 \\
\text { hebb }\end{array}$ & $\begin{array}{l}3 \\
\text { Id }\end{array}$ & \multicolumn{2}{|c|}{$\begin{array}{l}\text { mcfp aggregation } \\
\text { Combination func- } \\
\text { tion parameters } \\
\end{array}$} & \multicolumn{2}{|c|}{$\begin{array}{c}1 \\
\text { Alogistic }\end{array}$} & $\begin{array}{c}2 \\
\text { Hebb } \\
\mu \\
\end{array}$ & $\begin{array}{l}3 \\
\text { id }\end{array}$ & \multicolumn{3}{|c|}{\begin{tabular}{|c|}
$m s$ timing: \\
\\
\\
Speed factors \\
\end{tabular}} \\
\hline $\mathrm{wS}_{s}$ & & & 1 & $X_{1}$ & $\mathrm{ws}_{s}$ & & & & 1 & $X_{1}$ & $\mathrm{ws}_{s}$ & 0 \\
\hline $\mathrm{ss}_{s}$ & & & 1 & $X_{2}$ & $\mathrm{ss}_{s}$ & & & & 1 & $X_{2}$ & $\mathrm{ss}_{s}$ & 1 \\
\hline $\mathrm{srs}_{s}$ & & & 1 & $X_{3}$ & $\operatorname{srs}_{s}$ & & & & 1 & $X_{3}$ & $\operatorname{srs}_{s}$ & 1 \\
\hline $\mathrm{gs}_{b}$ & 1 & & & $X_{4}$ & $\mathrm{gs}_{b}$ & 18 & 0.62 & & & $X_{4}$ & $\mathrm{gs}_{b}$ & 0.01 \\
\hline $\mathrm{ps}_{g}$ & & & 1 & $X_{5}$ & $\mathrm{ps}_{g}$ & & & & 1 & $X_{5}$ & $\mathrm{ps}_{g}$ & 0.01 \\
\hline $\mathrm{es}_{g}$ & 1 & & & $X_{6}$ & $\mathrm{es}_{g}$ & 10 & 0.8 & & & $X_{6}$ & $\mathrm{es}_{g}$ & 0.01 \\
\hline$s s_{b}$ & & & 1 & $X_{7}$ & $s s_{b}$ & & & & 1 & $X_{7}$ & $s s_{b}$ & 0.2 \\
\hline $\operatorname{srs}_{b}$ & 1 & & & $X_{8}$ & $\operatorname{srs}_{b}$ & 10 & 0.3 & & & $X_{8}$ & $\operatorname{srs}_{b}$ & 0.2 \\
\hline $\mathrm{fs}_{b}$ & & & 1 & $X_{9}$ & $\mathrm{fs}_{b}$ & & & & 1 & $X_{9}$ & $\mathrm{fs}_{b}$ & 0.2 \\
\hline $\mathrm{ps}_{b}$ & 1 & & & $X_{10}$ & $\mathrm{ps}_{b}$ & 10 & 0.3 & & & $X_{10}$ & $\mathrm{ps}_{b}$ & 0.2 \\
\hline $\mathrm{es}_{b}$ & & & 1 & $X_{11}$ & $\mathrm{es}_{b}$ & & & & 1 & $X_{11}$ & $\mathrm{es}_{b}$ & 0.2 \\
\hline $\mathrm{bs}_{-}$ & 1 & & & $X_{12}$ & $\mathrm{bs}_{-}$ & 8 & 0.2 & & & $X_{12}$ & $\mathrm{bs}_{-}$ & 0.2 \\
\hline $\mathrm{bs}_{+}$ & 1 & & & $X_{13}$ & $\mathrm{bs}_{+}$ & 10 & 0.15 & & & $X_{13}$ & $\mathrm{bs}_{+}$ & 0.2 \\
\hline $\operatorname{accp}_{b}$ & 1 & & & $X_{14}$ & $\operatorname{accp}_{b}$ & 10 & 0.55 & & & $X_{14}$ & $\operatorname{accp}_{b}$ & 0.01 \\
\hline $\mathrm{aw}_{b}$ & 1 & & & $X_{15}$ & $a w_{b}$ & 10 & 0.55 & & & $X_{15}$ & $\mathrm{aw}_{b}$ & 0.01 \\
\hline $\mathrm{pr}_{b}$ & 1 & & & $X_{16}$ & $\mathrm{pr}_{b}$ & 10 & 0.55 & & & $X_{16}$ & $\mathrm{pr}_{b}$ & 0.01 \\
\hline$X_{17} \quad \mathrm{cs}_{\text {reapp }}$ & 1 & & & $X_{17}$ & $\mathrm{cs}_{\text {reapp }}$ & 10 & 0.44 & & & $X_{17}$ & $\mathrm{cs}_{\text {reapp }}$ & 0.28 \\
\hline$X_{18} \quad \mathbf{W}_{\mathrm{fs}_{b^{\prime}} \mathrm{cs}_{\text {reapp }}}$ & & 1 & & $X_{18}$ & $\mathbf{W}_{\mathrm{fs}_{b^{\prime}} \mathrm{cs}_{\text {reapp }}}$ & & & $X_{19}$ & & $X_{18}$ & $\mathbf{W}_{\mathrm{fs}_{b^{\prime}} \mathrm{cs}_{\text {reapp }}}$ & $X_{20}$ \\
\hline$X_{19} \quad \mathbf{M}_{\mathbf{W}_{\mathrm{fs}_{b}, \text { ss reapp }}}$ & 1 & & & $X_{19}$ & $\mathbf{M}_{\mathbf{W}_{\mathrm{fs}^{\prime}, \mathrm{s}_{\text {reapp }}}}$ & 10 & 0.85 & & & $X_{19}$ & $\mathbf{M}_{\mathbf{W}_{\mathrm{fs}_{b}, \mathrm{cs} \text { reapp }}}$ & 0.01 \\
\hline$X_{20} \quad \mathbf{H}_{\mathbf{W}_{\mathrm{fs}^{\prime}, \mathrm{cs} \text { reapp }}}$ & 1 & & & $X_{20}$ & $\mathbf{H}_{\mathbf{W}_{\mathrm{fs}_{b}, \text { cs reapp }}}$ & 10 & 0.8 & & & $X_{20}$ & $\mathbf{H}_{\mathbf{W}_{\mathrm{fs}_{b}, \mathrm{cs}_{\text {reapp }}}}$ & 0.01 \\
\hline
\end{tabular}

Box 2 Role matrices for aggregation and timing

In the meanwhile, gradual increase in the goal state $\mathrm{gs}_{b}$ takes place, since as a result of decrease in the activation level of $\mathrm{cs}_{\text {reapp }}$ the intensity of negative feelings $\mathrm{fs}_{b}$ increases which in turn activates the goal $\mathrm{gs}_{b}$ to undergo some therapy to regain cognitive control. Goal state $\mathrm{gs}_{b}$ activates the states that lay on the casual pathway describing undergoing MBCT therapy. Finally, when the execution state for goal (therapy) $\mathrm{es}_{g}$ gets enough momentum, as a result $\mathrm{cs}_{\text {reapp }}$ again gets activated. This means that the person has re-gained his cognitive reappraisal ability during execution of the therapy; however, at this stage the person still depends on the therapy for this. While the person is again becoming able to reappraise his or her emotions with a little help from the therapy, due to the achieved lower levels of $\mathrm{fs}_{b}$, the negative metaplasticity at the second reification level turns into positive metaplasticity by increasing the too low values of the two second-order states $\mathbf{M}_{\mathbf{W}_{\mathrm{fs}} \text {, csreapp }}$ and $\mathbf{H}_{\mathbf{W}_{\mathrm{fs}_{b}, \text { cs reapp }}}$ to higher values. Therefore, now a learning process takes place (that was blocked before by the negative metaplasticity due to low values of $\mathbf{M}_{\mathbf{W}_{\mathrm{fs}_{b}, \text { cs reapp }}}$ and $\mathbf{H}_{\mathbf{W}_{\mathrm{fs}_{b}} \text {, csreapp }}$ ), strengthening the connection from $\mathrm{fs}_{b}$ to $\mathrm{cs}_{\text {reap }}$. Due to the successful reappraisal, $\mathrm{fs}_{b}$ goes down, so it deactivates the goal $\mathrm{gs}_{b}$ to undergo therapy. This deactivates the therapy but the person is still able to reappraise his emotions effectively as before. It means that overall the connection has re-strengthened as a result of MBCT. This figure represents two phases of the subject's life. First phase is before his cognitive abilities are impaired by long-term stress where he regulates his emotions multiple times during that phase. The second phase is after the therapy when he's again 
able to use his cognitive abilities. The fluctuation in the graphs represent the cyclic nature of the stressful emotions that takes place repeatedly in cycles and are regulated accordingly.

This pattern is exactly as various researchers from cognitive and neuro-sciences have described in their articles as summarized in the background study in Sect. 2, for instance (Garcia 2002).

Figure 3 gives insight into the first and second-level reification states. The states represented in the figure are $\mathbf{W}_{\mathrm{fs}_{b}}$, $\mathrm{cs}_{\text {reapp }}, \mathbf{M}_{\mathbf{W}_{\mathrm{fs}_{b}} \text {, cs reapp }}, \mathbf{H}_{\mathbf{W}_{\mathrm{fs}_{b}} \text {, } \mathrm{cs}_{\text {reapp }}}$ which represent the adaptation of the indicated connection weight based on Hebbian learning, and the persistence factor and the speed factor for this adaptation, respectively. First it is shown that all these three states decrease because of the long-term stressors, but after activation of the therapy in the base model all three states again get higher values. It's worth noting here that all the states stay high even after the therapy gets deactivated in the base model. This shows that the person's wellbeing does not depend on the therapy anymore.

As already mentioned, $\mathbf{W}$ represent the Hebbian learning taking place at the connection indicated by $\mathrm{fs}_{b}, \mathrm{cs}_{\text {reapp }}$ in the base model. The speed factor and persistence of the $\mathbf{W}$ is handled by the $\mathbf{H}$ and $\mathbf{M}$-states respectively. In the Fig. 3 initially negative metaplasticity occurs (lower values of the second-order states) because of long-term stressors, so the learning gets blocked in a sense. Due to this, the connection $\mathrm{fs}_{b}, \mathrm{cs}_{\text {reapp }}$ gets weaker. But as the person undergoes MBCT, due to the positive effects that has on

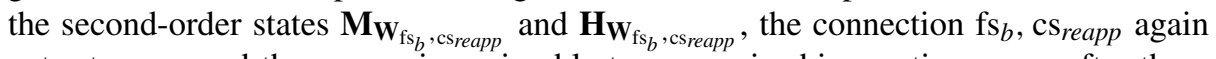
gets stronger and the person is again able to reappraise his emotions even after there is no more therapy. These results in Fig. 2 and Fig. 3 are completely in line with the findings from cognitive and neurosciences.

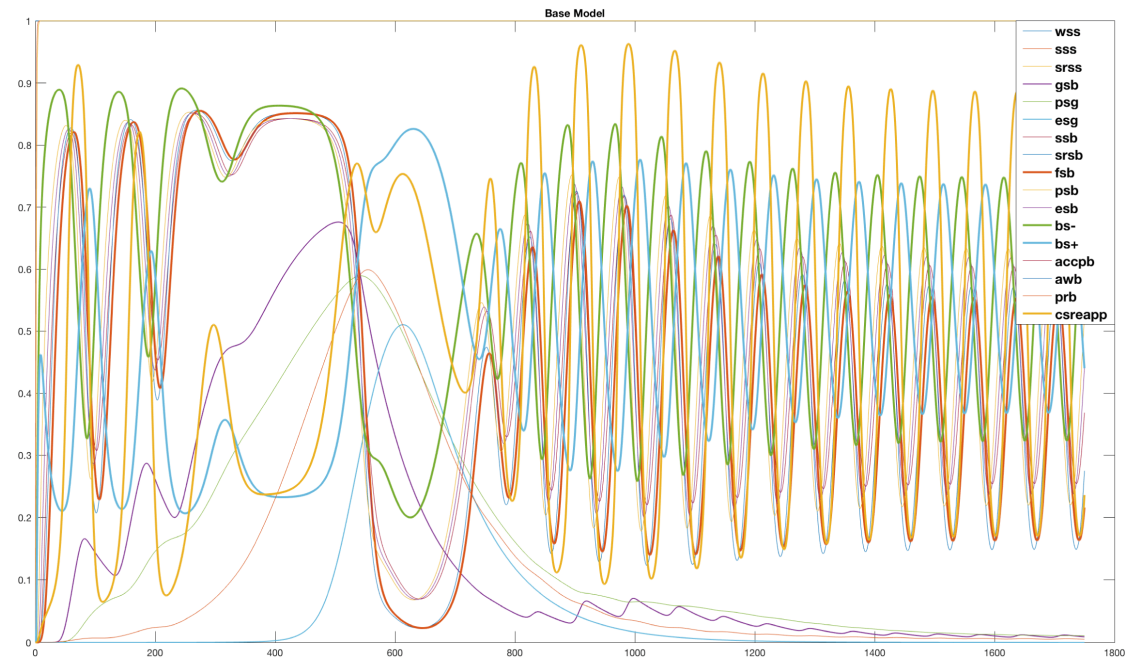

Fig. 2. Loss and gain of adaptive cognitive abilities as a result of long-term stressor (base level) 


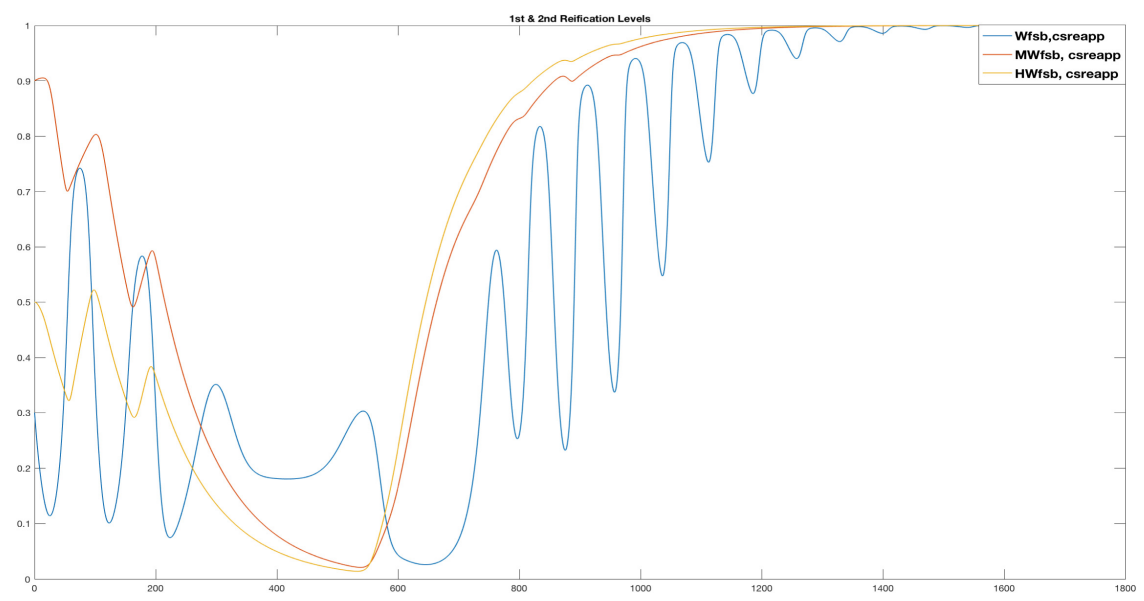

Fig. 3. First and second-order reification states for plasticity and metaplasticity over time

\section{Conclusion}

In this paper, the ideas on negative and positive metaplasticity from the (empirical) literature have been modeled and given a concrete shape in the form of simulations. On one hand, the multi-order adaptive causal network model presented in this paper gives a concrete shape to the abstract ideas, on the other hand it also highlights the consequences of long-term stressors along with the possible treatment for the psychological problems they cause. Findings from various neurological and psychological studies were brought together and presented in the form of a concrete computational model. Various therapies have already been modeled in work like (Ziabari and Treur 2019), but this paper considers therapy as a permanent treatment to cope with negative metaplasticity for the first time.

The paper also shows how the applicability scope of causal modeling, which has a long tradition in AI (e.g., see (Kuipers 1984; Kuipers and Kassirer 1983; Pearl 2009)), can substantially be widened by adding dynamics and (multi-order) adaptation to causal modeling. Doing this, causal modeling becomes applicable to modeling of dynamic and adaptive processes that otherwise would be out of reach for causal modeling.

The introduced second-order adaptive network model provides a good basis to develop a patient model (based on a virtual agent) for virtual training of therapists. In future, the author aims at extending the model by incorporating more neurological states for both the negative and positive metaplasticity and the neurological states involved in MBCT.

\section{References}

Allen, N.B., Blashki, G., Gullone, E.: Mindfulness-based psychotherapies: a review of conceptual foundations, empirical evidence and practical considerations. Aust. N. Z. J. Psychiatry 40(2), 285-294 (2006). https://doi.org/10.1111/j.1440-1614.2006.01794.x 
Butler, E.A., Egloff, B., Wlhelm, F.H., Smith, N.C., Erickson, E.A., Gross, J.J.: The social consequences of expressive suppression. Emotion 3(1), 48-67 (2003). https://doi.org/10.1037/15283542.3.1.48

Charles, S.T.: Strength and vulnerability integration: a model of emotional well-being across adulthood. Psychol. Bull. 136(6), 1068-1091 (2010). https://doi.org/10.1037/a0021232

Coffey, K.A., Hartman, M., Fredrickson, B.L.: Deconstructing mindfulness and constructing mental health: understanding mindfulness and its mechanisms of action. Mindfulness 1(4), 235-253 (2010). https://doi.org/10.1007/s12671-010-0033-2

Foster, T.C.: Involvement of hippocampal synaptic plasticity in age-related memory decline. Brain Res. Rev. 30(3), 236-249 (1999). https://doi.org/10.1016/s0165-0173(99)00017-x

Fuchs, E., Gould, E.: In vivo neurogenesis in the adult brain: regulation and functional implications. Eur. J. Neurosci. 12(7), 2211-2214 (2000)

Garcia, R.: Stress, metaplasticity, and antidepressants. Curr. Mol. Med. 2(7), 629-638 (2002). https://doi.org/10.2174/1566524023362023

Garland, E., Gaylord, S., Park, J.: The role of mindfulness in positive reappraisal. Explore 5(1), 37-44 (2009). https://doi.org/10.1016/j.explore.2008.10.001

Garland, E.L., Gaylord, S.A., Fredrickson, B.L.: Positive reappraisal mediates the stress-reductive effects of mindfulness: an upward spiral process. Mindfulness 2(1), 59-67 (2011). https://doi. org/10.1007/s12671-011-0043-8

Gross, J.J., John, O.P.: Individual differences in two emotion regulation processes: implications for affect, relationships, and well-being. J. Pers. Soc. Psychol. 85(2), 348-362 (2003). https:// doi.org/10.1037/0022-3514.85.2.348

Jha, A.P., Krompinger, J., Baime, M.J.: Mindfulness training modifies subsystems of attention. Cogn. Affect. Behav. Neurosci. 7(2), 109-119 (2007)

John, O.P., Gross, J.J.: Healthy and unhealthy emotion regulation: personality processes, individual differences, and life span development. J. Pers. 72(6), 1301-1334 (2004). https://doi.org/10. 1111/j.1467-6494.2004.00298.x

Kabat-Zinn, J.: Full Catastrophe Living: Using the Wisdom of Your Body and Mind to Face Stress, Pain and Illness, 1st edn. Delta, New York (1990)

Kim, J.J., Yoon, K.S.: Stress: metaplastic effects in the hippocampus. Trends Neurosci. 21(12), 505-509 (1998). https://doi.org/10.1016/s0166-2236(98)01322-8

Kuipers, B.: Commonsense reasoning about causality: deriving behavior from structure. Artif. Intell. 24, 169-203 (1984). https://doi.org/10.1016/0004-3702(84)90039-0

Kuipers, B., Kassirer, J.P.: How to discover a knowledge representation for casual reasoning by studying an expert pysician. In: Proceedings of the IJCAI 1983 (1983)

Lupien, S.J., et al.: Stress-induced declarative memory impairment in healthy elderly subjects: relationship to cortisol reactivity. J. Clin. Endocrinol. Metab. 82(7), 2070-2075 (1997). https:// doi.org/10.1210/jcem.82.7.4075

Mazure, C.M., Maciejewski, P.K., Jacobs, S.C., Bruce, M.L.: Stressful life events interacting with cognitive/personality styles to predict late-onset major depression. Am. J. Geriatr. Psychiatry 10(3), 297-304 (2002)

Mizoguchi, K., Yuzurihara, M., Ishige, A., Sasaki, H., Chui, D.H., Tabira, T.: Chronic stress induces impairment of spatial working memory because of prefrontal dopaminergic dysfunction. J. Neurosci. 20(4), 1568-1574 (2000)

Mocchetti, I., Spiga, G., Hayes, V., Isackson, P., Colangelo, A.: Glucocorticoids differentially increase nerve growth factor and basic fibroblast growth factor expression in the rat brain. J. Neurosci. 16(6), 2141-2148 (1996). https://doi.org/10.1523/JNEUROSCI.16-06-02141.1996

Mohammadi Ziabari, S.S., Treur, J.: Cognitive modeling of mindfulness therapy by autogenic training. In: Satapathy, S.C., Bhateja, V., Somanah, R., Yang, X.-S., Senkerik, R. (eds.) Information Systems Design and Intelligent Applications. AISC, vol. 863, pp. 53-66. Springer, Singapore (2019). https://doi.org/10.1007/978-981-13-3338-5_6 
Pearl, J.: Causality. Cambridge University Press, Cambridge (2009)

Sapolsky, R.M.: Glucocorticoids, stress, and their adverse neurological effects: relevance to aging. Exp. Gerontol. 34(6), 721-732 (1999). https://doi.org/10.1016/S0531-5565(99)00047-9

Smith, M.A., Makino, S., Kvetnansky, R., Post, R.M.: Stress and glucocorticoids affect the expression of brain-derived neurotrophic factor and neurotrophin-3 mRNAs in the hippocampus. J. Neurosci. 15(3), 1768-1777 (1995)

Tennant, C.: Work-related stress and depressive disorders. J. Psychosom. Res. 51(5), 697-704 (2001). https://doi.org/10.1016/S0022-3999(01)00255-0

Treur, J.: Network-Oriented Modeling: Addressing Complexity of Cognitive, Affective and Social Interactions. Springer, Cham (2016). https://doi.org/10.1007/978-3-319-45213-5

Treur, J.: Network-Oriented Modeling for Adaptive Networks: Designing Higher-Order Adaptive Biological. Mental and Social Network Models. Springer, Cham (2020). https://doi.org/10. 1007/978-3-030-31445-3

Troy, A.S., Shallcross, A.J., Davis, T.S., Mauss, I.B.: History of mindfulness-based cognitive therapy is associated with increased cognitive reappraisal ability. Mindfulness 4(3), 213-222 (2013). https://doi.org/10.1007/s12671-012-0114-5

Ullah, N., Treur, J.: The choice between bad and worse: a cognitive agent model for desire regulation under stress. In: Baldoni, M., Dastani, M., Liao, B., Sakurai, Y., Zalila Wenkstern, R. (eds.) PRIMA 2019. LNCS (LNAI), vol. 11873, pp. 496-504. Springer, Cham (2019). https:// doi.org/10.1007/978-3-030-33792-6_34

Ullah, N., Treur, J.: Better late than never: a multilayer network model using metaplasticity for emotion regulation strategies. In: Cherifi, H., Gaito, S., Mendes, J.F., Moro, E., Rocha, L.M. (eds.) COMPLEX NETWORKS 2019. SCI, vol. 882, pp. 697-708. Springer, Cham (2020). https://doi.org/10.1007/978-3-030-36683-4_56

Verhaeghen, P.: Cognitive processes and ageing. In: Stuart-Hamilton, I. (ed.) An Introduction to Gerontology, pp. 159-193. Cambridge University Press, Cambridge (2011). https://doi.org/10. 1017/CBO9780511973697.006 